Nervenarzt 2009 $\cdot 80: 873-874$

DOI 10.1007/s00115-009-2813-2

Online publiziert: 12. August 2009

(c) Springer Medizin Verlag 2009

\author{
T. Brandt \\ Institut für Klinische Neurowissenschaften, Klinikum der LMU München, \\ Campus Großhadern, München
}

\title{
Aus dem Gleichgewicht
}

\section{Leitsymptom Schwindel}

Schwindel ist eines der häufigsten Leitsymptome in der Medizin mit einer Lebenszeitprävalenz von etwa 30\%. Ähnlich wie beim häufigen Leitsymptom „Kopfschmerz " können interdisziplinär ausgebildete Spezialisten die verschiedenen Schwindelformen in der Regel allein nach sorgfältiger Anamnese und körperlicher Untersuchung, ohne apparative Zusatzuntersuchung zuverlässig diagnostizieren und wirkungsvoll therapieren. Dennoch besteht national und international eine Unter- und Fehlversorgung dieser Patienten. Dies führt dazu, dass Betroffene nacheinander Ärzte unterschiedlicher Disziplinen (Neurologie, HNO, Innere Medizin, Orthopädie, Psychiatrie) aufsuchen mit unnötiger apparativer Diagnostik, Zuordnung zu falschen Diagnosen und mangelhafter Therapie. Häufig resultieren daraus lange Phasen von Arbeits-, Erwerbs- und Berufsunfähigkeit mit beträchtlichen psychosozialen und ökonomischen Folgen [1].

\section{( ) National und international besteht eine Unter- und Fehlversorgung der "Schwindel-Patienten"}

Die Einrichtung eines integrierten Forschungs- und Behandlungszentrums für Schwindel, Gleichgewichts- und Okulomotorikstörungen (IFB ${ }^{\mathrm{LMU}}$ ) durch das Bundesministerium für Bildung und Forschung (BMBF) in München ist international einmalig. Entsprechend der Ausschreibung möchte das BMBF "geeignete Initiativen an den medizinischen Fakultäten und Universitätsklinika im Rahmen des Programms der Bundesregie- rung, Gesundheitsforschung: Forschung für den Menschen' unterstützen. Vorgesehen ist die Förderung innovativer Forschungs- und Behandlungszentren, in denen die klinische Spitzenforschung zu spezifischen Krankheitsgebieten durch ein integratives Miteinander von klinischer Forschung und Krankenversorgung auf höchstem Niveau gefördert wird. Mit den integrierten Forschungs- und Behandlungszentren soll die wissenschaftliche Wettbewerbsfähigkeit erhöht, eine stärkere Nutzung der vorhandenen Ressourcen für leistungsstarke Bereiche und zugleich ein effektiver Transfer von Forschungsergebnissen in das Versorgungsgeschehen befördert werden." Das wissenschaftliche und strukturelle Gesamtkonzept des $\mathrm{IFB}^{\mathrm{LMU}}$ wird in diesem Leitthemenheft dargestellt [1].

Vier weitere Beiträge behandeln klinisch sehr relevante, aber häufig vernachlässigte Themen wie die Epidemiologie von Schwindelerkrankungen (Hannelore Neuhauser [5]), die vestibuläre Migräne (Thomas Lempert [4]), die kindlichen Schwindelformen (Klaus Jahn [3]) sowie somatoforme Schwindelsyndrome (Annegret Eckhardt-Henn und Mitarbeiter [2]).

Epidemiologische Daten zu Schwindelerkrankungen ergaben interessante Assoziationen zwischen vestibulärem Schwindel und Migräne: $z$. B. von Migräne mit benignem paroxysmalem Lagerungsschwindel und Morbus Menière, wobei die pathophysiologischen Mechanismen noch unklar sind [5]. Die vestibuläre Migräne wird erst seit den 1980er Jahren zunehmend als eigenständiges Syndrom diagnostiziert und therapiert [4]. Sie ist die 
häufigste Ursache spontaner Schwindelepisoden mit einer variablen Dauer von Sekunden bis Tagen, häufig auch ohne Kopfschmerzen. Umso wichtiger sind klare diagnostische Kriterien und Therapiestudien, wie von Thomas Lempert diskutiert. Migräneassoziierte Schwindelformen (der benigne paroxysmale Schwindel des Kindesalters und die vestibuläre Migräne) machen etwa 50\% der Diagnosen der Schwindelsyndrome im Kindesalter aus [3]. Schwindel ist auch bei Kindern nicht selten. Alle bei Erwachsenen bekannten Syndrome kommen auch bei Kindern vor; häufiger als bei Erwachsenen sind peripher-vestibuläre Erkrankungen im Rahmen von infektiösen oder parainfektiösen Labyrinthiden sowie zentral-vestibuläre Syndrome bei Kleinhirnund Hirnstammtumoren [3]. Auch die Bewegungskrankheit der Kinder ist besonders im Alter zwischen 4 und 10 Jahren beim Transport in Fahrzeugen ein häufiges Problem.

Der somatoforme Schwindel ist der Oberbegriff unterschiedlicher Subgruppen psychischer und psychosomatischer Störungen mit dem Leitsymptom Schwindel, vor allem des phobischen Schwankschwindels. Annegret Eckhardt-Henn beschreibt ein pathogenetisches Model des primären und des sekundären somatoformen Schwindels [2].

\section{Korrespondenzadresse}

\section{Prof. Dr. h.c. T. Brandt FRCP}

Institut für Klinische Neurowissenschaften, Hertie Senior Forschungsprofessur, Klinikum der LMU München,

Campus Großhadern

Marchioninistraße 15, 81377 München

thomas.brandt@med.uni-muenchen.de

\section{Literatur}

1. Brandt T, Zwergal A, Jahn K, Strupp M (2009) Integriertes Forschungs- und Behandlungszentrum für Schwindel, Gleichgewichts- und Okulomotorikstörungen (IFB $\left.{ }^{\mathrm{LMU}}\right)$. Der Nervenarzt ((Heft 08/09))

2. Eckhardt-Henn A, Tschan R, Best C, Dieterich M (2009) Somatoforme Schwindelsyndrome. Der Nervenarzt

3. Jahn K (2009) Kindliche Schwindelformen. Klinik, Verlauf, Therapie. Der Nervenarzt

4. Lempert T (2009) Vestibuläre Migräne. Der Nervenarzt

5. Neuhauser HK (2009) Epidemiologie von Schwindelerkrankungen. Der Nervenarzt

\section{EU-Forschungsprojekt CEDAR}

Aufruf zur Studienteilnahme

Forschung zu klinischer Entscheidungsfindung fand bislang vor allem im Bereich kurzfristiger körperlicher Erkrankungen statt. In Bezug auf Entscheidungsprozesse und damit verbundene Behandlungsergebnisse im Rahmen von längerfristigen und speziell psychischen Erkrankungen besteht hingegen eine Forschungslücke.

Daher begann im April 2009 unter Leitung der Klinik für Psychiatrie und Psychotherapie II der Universität Ulm die im 7. EU-Forschungsrahmenprogramm geförderte multizentrische Studie "Clinical decision making and outcome in routine care for people with severe mental illness" (Akronym: CEDAR). Gemeinsam mit kooperierenden universitären Zentren in London, Neapel, Zürich, Debrecen (Ungarn) und Aalborg (Dänemark) untersucht das Ulmer Forschungsteam um Dr. Bernd Puschner und Prof. Dr. Thomas Becker in dieser prospektiven Beobachtungsstudie die Art und Weise der Entscheidungsfindung von Patienten und Behandlern im Verlauf einer psychischen Erkrankung. Mittels wiederholter, jeweils in Monatsabständen erfolgender Befragungen von Patienten und ihren Behandlern über den Beobachtungszeitraum von einem Jahr hinweg werden die kurz-, mittel- und langfristigen Effekte verschiedener Varianten klinischer Entscheidungsfindung auf BehandlungsOutcomes wie etwa Symptomschwere oder Lebensqualität untersucht. Hierdurch wird es möglich, Merkmale klinischer Entscheidungsfindung zu identifizieren, die mit guten Behandlungsergebnissen bei Menschen mit schweren psychischen Erkrankungen in Zusammenhang stehen. Daraus sollen Empfehlungen für eine gute klinische Praxis formuliert werden. Es wird insgesamt erwartet, dass der Forschungsstand zu klinischer Entscheidungsfindung durch CEDAR entscheidend ergänzt werden kann.

Aufruf zur Studienteilnahme an niedergelassene Psychiater, Psychologen oder Sozialpädagogen

Im Rahmen von CEDAR wird derzeit an der Entwicklung eines Erhebungsinstrumentes gearbeitet, welches die im Zeitverlauf auftretenden Entscheidungsprozesse von psychisch erkrankten Patienten und ihren Behandlern adäquat abbildet. Dieses Instrument soll Items zum Entscheidungsstil von Patienten und Behandlern, zum konkreten Ablauf von Entscheidungen in der Patient-Behandler-Interaktion sowie zur Beteiligung an und Zufriedenheit mit getroffenen Entscheidungen enthalten. Im Oktober 2009 wird die eigentliche Hauptstudie beginnen. Dafür suchen wir mit psychisch kranken Menschen arbeitende niedergelassene (oder in Psychiatrischen Institutsambulanzen arbeitende) Ärzte für Psychiatrie und Psychotherapie (oder Nervenärzte), Psychologen oder Sozialarbeiter, die bereit sind, über ein Jahr hinweg einmal monatlich (13 Befragungszeitpunkte) an einer schriftlichen Befragung teilzunehmen. Die zu den wiederholten Messzeitpunkten gestellten Fragen beziehen sich schwerpunktmäßig auf die Treffen mit einem - und zwar immer demselben - Patienten. Die Fragebögen können entweder online am PC oder in Papierform ausgefüllt werden, wobei die Beantwortung zu jedem Befragungszeitpunkt etwa 20 Minuten dauern wird. Eine Ausnahme bilden die Anfangs- und Endbefragungen, die jeweils etwa eine halbe Stunde in Anspruch nehmen werden. Für die Studienteilnahme werden Büchergutscheine im Gesamtwert von 50 Euro sowie CME-Punkte vergeben.

Wenn Sie an CEDAR teilnehmen möchten oder weitergehende Fragen haben, wenden Sie sich bitte an:

\section{Dipl.-Soz. Katrin Arnold}

Klinik für Psychiatrie und Psychotherapie II der Universität UIm

Bezirkskrankenhaus Günzburg Ludwig-Heilmeyer-Str. 2

89312 Günzburg

Telefon: 08221-962864

Fax: 08221-9628160

E-mail: katrin.arnold@bkh-guenzburg.de

Quelle: Klinik für Psychiatrie und Psychotherapie II, Universität UIm 